\title{
Newborn screening for HLA markers associated with IDDM: Diabetes Autoimmunity Study in the Young (DAISY)
}

\author{
M. Rewers ${ }^{1}$, T.L. Bugawan ${ }^{2}$, J. M. Norris ${ }^{1}$, A. Blair ${ }^{2}$, B. Beaty ${ }^{1}$, M.Hoffman ${ }^{1}$, R.S.McDuffie, Jr. ${ }^{4}$, R. F.Hamman ${ }^{1}$, \\ G. Klingensmith ${ }^{3}$, G.S. Eisenbarth ${ }^{3}$, H. A. Erlich ${ }^{2}$ \\ ${ }^{1}$ Department of Preventive Medicine and Biometrics, University of Colorado, School of Medicine, Denver, Colorado, USA \\ ${ }^{2}$ Human Genetics Department, Roche Molecular Systems, Inc., Alameda, California, USA \\ ${ }^{3}$ Barbara Davis Center for Childhood Diabetes, Denver, Colorado, USA \\ ${ }^{4}$ Department of Obstetrics and Gynecology, Kaiser Permanente and St. Joseph's Hospital, Denver, Colorado, USA
}

\begin{abstract}
Summary Autoimmunity causing insulin-dependent diabetes mellitus (IDDM) begins in early childhood due to interactions between genes and unknown environmental factors that may be identified through follow-up of a large cohort of genetically susceptible children. Such a cohort has been established using a simple and rapid cord blood screening for HLA alleles. The DRB1 and DQB1 second exon sequences were co-amplified using the polymerase chain reaction and hybridized with single and pooled sequence-specific oligonucleotide probes. Four individual probes were used to detect the susceptibility alleles DRB1*03, DRB1*04, and DQB1*0302 as well as the usually protective DRB1*15/16 (DR2) alleles. In addition, pooled probes allow the distinction of DR3/3 from the DR3/x genotype (where $x$ is neither DR2, 3, nor 4) and DR4/4 from DR4/x. Among 5000 newborns from the general Denver population, we
\end{abstract}

have found the high-risk genotype (DRB1*03/ DRB1*04, DQB1*0302) to be present in $2.4 \%$ of non-Hispanic whites, $2.8 \%$ of Hispanics, and $1.6 \%$ of African Americans. The moderate-risk genotypes (DRB $1 * 04, \quad$ DQB $1 * 0302 / \mathrm{DRB} 1 * 04, \quad \mathrm{DQB} 1 * 0302$, DRB $1 * 04, \mathrm{DQB} 1 * 0302 / \mathrm{x}$, or DRB $1 * 03 / \mathrm{DRB} 1 * 03$ ) are present in $17 \%$ of American non-Hispanic whites, $24 \%$ of Hispanics and in $10 \%$ of African Americans. These results demonstrate the feasibility of a large-scale newborn screening for genes associated with IDDM. The ultimate role for such a screening in future routine prediction and prevention of IDDM will depend on the availability of an effective and acceptable form of clinical intervention. [Diabetologia 1996) 39: 807-812]

Keywords Insulin-dependent diabetes mellitus, HLA class II alleles, newborn screening, autoimmunity.
Insulin-dependent diabetes mellitus (IDDM) is caused by interactions between genetic and environmental factors [1]. While a number of genetic loci have been associated and linked with IDDM [2-10], the environmental causes remain to be determined. To accomplish this, a large cohort of genetically

\section{Received: 21 July 1995 and in revised form: 7 December 1995}

Corresponding author: Dr. M. Rewers, Department of Preventive Medicine and Biometrics, University of Colorado, School of Medicine, $4200 \mathrm{E}$ 9th Avenue, C-245, Denver, CO 80262 USA

Abbreviations: IDDM, Insulin-dependent diabetes mellitus; PCR, polymerase chain reaction; SSO, sequence-specific oligonucleotide; DAISY, Diabetes Autoimmunity Study in the Young; HRP, horseradish peroxidase; TMB, tetramethyl benzidine; SSPE, saline-sodium phosphate-EDTA susceptible infants needs to be followed with frequent measurements of candidate exposures from birth into early childhood when prediabetic autoimmunity often develops [11]. This report describes genetic screening based on HLA-DR/DQ typing of cord blood samples to identify a cohort of newborns who are genetically susceptible to IDDM and can be followed for development of autoimmunity and diabetes.

The HLA region on chromosome $6 \mathrm{p} 21$, the major genetic determinant of IDDM, accounts for about one-half of the genetic susceptibility to IDDM [7, 8]. No other single genetic region confers a comparable risk. In Caucasians, HLA class II alleles DRB1*04, DRB $1 * 03$ and DQB1*0302 confer susceptibility while DQB $1 * 0602$ is protective $[2-5,12-14]$. Largescale population screening for these markers has 
become possible due to simplification of HLA class II typing, based on polymerase chain reaction (PCR) amplification and sequence-specific oligonucleotide (SSO) probe hybridization [15, 16]. We describe such a general population newborn screening for IDDM-associated HLA markers, initiated to better define the natural history of prediabetic autoimmunity and its environmental causes. In addition to technical and logistic feasibility, parental acceptance of such a screening was evaluated in a multiethnic population.

\section{Subjects and methods}

Study population. Between February 1994 and June 1995, the Diabetes Autoimmunity Study in the Young (DAISY) screened 5045 newborns from the general population of Denver, Colorado, USA to identify and follow-up for development of beta-cell autoimmunity those at genetically increased IDDM risk. The aims of the follow-up include assessment of age- and genotype-specific incidence of autoimmunity, evaluation of its candidate environmental causes as well as determination of promoters of progression from autoimmunity to diabetes. All newborns born at St. Joseph's Hospital in Denver were eligible for the screening; however, newborns with severe congenital abnormalities or extreme prematurity were excluded. Delivery is often complicated in such cases and compliance of medical personnel with this research protocol was expected to be low. In addition, participation in the follow-up was deemed to be of low priority to the affected families.

The population screened is representative of the general newborn population of the Denver Metropolitan Area and includes children classified by their mothers as non-Hispanic white $(61 \%)$, Hispanic (22\%), African American (9\%), Asian American $(2 \%)$ or other $(6 \%)$. Approximately $75 \%$ of the newborns are members of the Kaiser Permanente Colorado Heath Maintenance Organization, while the others represent a mix of Medicaid, uninsured and privately insured populations.

Collection of cord blood samples. After the umbilical cord was wiped with a sterile gauze a routine blood sample was obtained for blood group typing and a second sterile tube containing $15 \%$ EDTA was filled with approximately $10 \mathrm{ml}$ of blood for DAISY screening. The additional DAISY sample was not obtained for $15 \%$ of the newborns during the study period. Prior to hospital discharge, a study recruiter attempted to meet with the mother of each child for whom the screening sample was obtained ( $n=5817)$, to present the study and to obtain written informed consent to screening. The informed consent process emphasizes that the screening is for research purposes only and that IDDM is expected to develop, by the age of 20 years, in only 1 in 15 of children with the highest risk genes, and much less frequently in children with moderate-risk genotypes. It included information that the results of this screening, if interpreted incorrectly, may affect the child's ability to get medical or life insurance in the future. The informed consent form is available on request from the authors.

Informed consent to screening was obtained for 5053 $(87 \%)$ of the newborns. We were unable to contact parents of $7 \%$ of the eligible children, usually due to early hospital discharge. While $94 \%$ of those contacted agreed to screening, $6 \%(n=333)$ of parents who were asked to participate refused the screening. Of those, 134 completed a refuser interview, which indicated that $71 \%$ of the families did not want to be involved in any kind of research, $9 \%$ did not want to know the results and $7 \%$ feared that results would affect future health insurance. The refusal rate was similar among members of Kaiser Permanente (5.8\%) and among privately insured/uninsured $(6.3 \%)$ and did not differ by ethnicity. Blood samples of newborns for whom informed consent could not be obtained were destroyed. All study protocols were in accordance with the principles of the Declaration of Helsinki and approved by the Colorado Multiple Institutional Review Board.

Sample preparation. The whole blood was aliquoted in two 0.5 ml samples: 1) the primary typing sample remained at room temperature for a period of 1-14 days before DNA preparation; and 2) the blinded duplicate quality control sample was refrigerated. Samples were sent in weekly batches to the HLA typing laboratory at Roche Molecular Systems, Inc. (Alameda, Calif., USA). Whole blood (15-25 $\mu \mathrm{l})$ was added to $1.0 \mathrm{ml}$ of RMS blood dilution reagent, containing tris- $\mathrm{HCl}$, detergent, proteinase $\mathbf{K}$, and sodium azide. The diluted blood was mixed by vortexing briefly and then incubated at $100^{\circ} \mathrm{C}$ for $30 \mathrm{~min}$ in a dry heat temperature block. The samples were then centrifuged at $15000 \mathrm{rev} / \mathrm{min}$ for $2 \mathrm{~min}$ and the extract was refrigerated until PCR amplification.

PCR amplification. Forty microlitres of the extract was added to $60 \mu \mathrm{l}$ of standard PCR reaction mix containing 10X PCR Buffer, $100 \mu \mathrm{mol} / \mathrm{l}$ each of dATP, dCTP, dGTP, and dTTP, $30 \mathrm{pmol} / \mathrm{l}$ of each amplification primer, 5 units Taq DNA polymerase and $10 \%$ glycerol. Two sets of primers were used to co-amplify the DRB1 locus: GH46 and AB60 [16] and the DQB1 locus: DB130 and GH29 [15]. The amplification was carried out in a GeneAmp PCR System 9600 thermal cycler (Perkin-Elmer, Norwalk, CN) using a three-step temperature cycle: denaturation at $95^{\circ} \mathrm{C}$ for $20 \mathrm{~s}$, annealing at $55^{\circ} \mathrm{C}$ for $1 \mathrm{~min}$, and extension at $72^{\circ} \mathrm{C}$ for $30 \mathrm{~s}$, for 37 cycles. A 'no DNA' control was always included in the amplification to check for PCR product carry-over. A control blood sample was prepared as described above and subject to the same thermal cycling profile without the components of the PCR mix and spotted on the membrane. This sample served as a control for background on the DNA blots. The efficiency of the amplification was monitored initially by examining $3 \mu \mathrm{l}$ of the PCR product on a $3 \%$ Nusieve (FMC Bioproducts, Rockland, ME), $1 \%$ agarose gel.

Dot-blot of PCR product. Ninety microlitres of amplified DNA sample was denatured in $260 \mu \mathrm{l}$ of $0.4 \mathrm{~N} \mathrm{NAOH}$, $25 \mathrm{mmol} / 1 \mathrm{EDTA}$ for $10 \mathrm{~min}$ at room temperature (six replicate membranes were made). Denatured DNA sample (50 $\mu \mathrm{l})$ was applied to each membrane using a dot-blot apparatus and ultraviolet cross-linked using a Stratalinker (Stratagene, La Jolla, CA) at $500 \mathrm{~mJ} / \mathrm{cm}^{2}$.

Hybridization. The membranes were hybridized with either biotinylated or horseradish peroxidase (HRP)-labelled probes ( 2 pmol probe per $\mathrm{ml}$ of hybridization solution). Table 1 summarizes the SSO probes used in this screening programme and their reactivity patterns. The probes GH104, GH59, GH56, Pool 1, and Pool 2 were hybridized in $2.0 \times$ salinesodium phosphate-EDTA (SSPE) $+0.5 \%$ SDS at $42^{\circ} \mathrm{C}$ for $30 \mathrm{~min}$ and washed in $0.2 \times \mathrm{SSPE}$ at $42^{\circ} \mathrm{C}$ for $15 \mathrm{~min}$. Probe CRX49 was washed in $1.0 \times$ SSPE $+0.1 \%$ SDS at $42^{\circ} \mathrm{C}$. Probe DRB302 was washed in $0.1 \times \mathrm{SSPE}+0.1 \% \mathrm{SDS}$ at $50^{\circ} \mathrm{C}$. Probe DB272 was hybridized in $1 \times$ SSPE $+0.5 \%$ SDS for $30 \mathrm{~min}$ at $50^{\circ} \mathrm{C}$ and washed in $0.1 \times \mathrm{SSPE}+0.1 \%$ SDS for 10 min. Probe DB503 was hybridized in $3.0 \times \mathrm{SSPE}+0.5 \%$ SDS at $50^{\circ} \mathrm{C}$ and washed in $1.0 \times \mathrm{SSPE}+0.1 \%$ SDS at $50^{\circ} \mathrm{C}$. Hybridization of the biotinylated probes was detected by incubation with streptavidin-HRP conjugate and subsequently 
Table 1. SSO probe reactivity patterns

\begin{tabular}{|c|c|c|c|c|c|c|c|c|c|c|}
\hline $\begin{array}{l}\text { IDDM } \\
\text { risk }\end{array}$ & HLA-DR & $\begin{array}{l}\text { DR3 } \\
\text { DRB302 } \\
\text { (KGR) }\end{array}$ & $\begin{array}{l}\text { DR4 } \\
\text { GH59 } \\
\text { (VH) }\end{array}$ & $\begin{array}{l}\text { DR1,8,9,10,12 } \\
\text { Pool 1 } 1^{\mathrm{a}}\end{array}$ & $\begin{array}{l}\text { DR } 4,5,6,7,9 \\
\text { Pool } 2^{\mathrm{b}}\end{array}$ & $\begin{array}{l}\text { DR3,5,6 } \\
\text { GH56 } \\
\text { (YSTS) }\end{array}$ & $\begin{array}{l}\text { DR7 } \\
\text { CRX49 } \\
(\text { GYK) }\end{array}$ & $\begin{array}{l}\text { DR2 } \\
\text { GH104 } \\
\text { (WPR) }\end{array}$ & $\begin{array}{l}\text { DQB1*0302 } \\
\text { DB272 } \\
\text { (LGPPA) }\end{array}$ & $\begin{array}{l}\text { DQB1 } \\
\text { DB503 } \\
\text { (All) }\end{array}$ \\
\hline$\overline{\text { High }}$ & $3 / 4$ & + & + & - & + & + & - & - & + & + \\
\hline Moderate & $4 / 4$ & - & + & - & + & - & - & - & + & + \\
\hline Moderate & $4 / x$ & - & + & + or - & + & - & + or - & - & + & + \\
\hline Moderate & $3 / 3$ & + & - & - & - & + & - & - & - & + \\
\hline Low & $3 / 4$ & + & + & - & + & + & - & - & - & + \\
\hline Low & $4 / 4$ & - & + & - & + & - & - & - & - & + \\
\hline Low & $4 / x$ & - & + & + or - & + & + or - & + or - & - & - & + \\
\hline Low & $4 / 2$ & - & + & - & + & - & - & + & - & + \\
\hline Low & $4 / 2$ & - & + & - & + & - & - & + & + & + \\
\hline Low & $3 / 2$ & + & - & - & - & + & - & + & - & + \\
\hline Low & $3 / x$ & + & - & + or - & + or - & + & + or - & - & - & + \\
\hline Low & $2 / 2$ & - & - & - & - & - & - & + & - & + \\
\hline Low & $\mathrm{x} / \mathrm{x}$ & - & - & + or - & + or - & + or - & + or - & - & - & + \\
\hline
\end{tabular}

Sequence motifs (epitopes) recognized by the probes are shown in parentheses

${ }^{a}$ Pool 1 contains four probes (DB495, GH102, GH111, CRX24) and identifies types DR1, DR8, DR12, DR9, and DR10 b Pool 2 contains six probes (CRX35, CRX06, CRX68, CRX63, CRX15) and identifies DR5 and DR6 as well as DRB4 (DR4, DR7 and DR9)

${ }^{c}$ Control DQB1 probe added to confirm amplification of DQB1

$\mathrm{x}$, Neither DR2, 3 nor 4

Table 2. Population frequencies [\%] of HLA gene markers associated with IDDM, DAISY 1994-1995, Denver, Colorado, USA

\begin{tabular}{|c|c|c|c|c|c|c|}
\hline $\begin{array}{l}\text { IDDM risk category } \\
\text { HLA-DR, DQ } \\
n\end{array}$ & $\begin{array}{l}\text { Non-Hispanic } \\
\text { whites } \\
3063\end{array}$ & $\begin{array}{l}\text { Hispanics } \\
1125\end{array}$ & $\begin{array}{l}\text { African } \\
\text { Americans } \\
431\end{array}$ & $\begin{array}{l}\text { Asian } \\
\text { Americans } \\
99\end{array}$ & $\begin{array}{l}\text { Biracial and } \\
\text { others } \\
327\end{array}$ & $\begin{array}{l}\text { All } \\
5045\end{array}$ \\
\hline $\begin{array}{l}\text { High risk }(1: 16) \\
\text { DR3/4, DQB1*0302 }\end{array}$ & 2.42 & 2.76 & 1.62 & - & 1.83 & 2.34 \\
\hline $\begin{array}{l}\text { Moderate risk }(1: 75-1: 230) \\
\text { DRx } / 4, \text { DQB } 1 * 0302 \\
\text { DR4/4, DQB } 1 * 0302 \\
\text { DR3/3 }\end{array}$ & $\begin{array}{r}16.69 \\
11.43 \\
3.59 \\
1.67\end{array}$ & $\begin{array}{r}24.44 \\
19.91 \\
4.00 \\
0.53\end{array}$ & $\begin{array}{l}9.74 \\
7.19 \\
0.46 \\
2.09\end{array}$ & $\begin{array}{l}9.09 \\
7.07 \\
2.02 \\
-\end{array}$ & $\begin{array}{r}16.82 \\
13.15 \\
2.45 \\
1.22\end{array}$ & $\begin{array}{r}17.67 \\
12.97 \\
3.31 \\
1.39\end{array}$ \\
\hline $\begin{array}{l}\text { Low risk }(\leq 1: 300) \\
\text { DR3/x } \\
\text { DR3/4, DQB1 not } * 0302 \\
\text { DRx/4, DQB1 not } * 0302 \\
\text { DR4/4, DQB1 not } * 0302 \\
\text { DR2/4, DQB1*0302 } \\
\text { DR2/4, DQB1 not } * 0302 \\
\text { DR2/3 } \\
\text { DRx/x, } 2 / \times \text { or } 2 / 2\end{array}$ & $\begin{array}{r}80.90 \\
13.06 \\
1.50 \\
7.31 \\
0.49 \\
3.43 \\
2.19 \\
4.18 \\
48.74\end{array}$ & $\begin{array}{r}72.81 \\
9.78 \\
0.44 \\
3.82 \\
0.18 \\
4.09 \\
1.42 \\
1.96 \\
51.12\end{array}$ & $\begin{array}{r}88.63 \\
14.85 \\
0.70 \\
2.78 \\
- \\
1.39 \\
0.93 \\
5.10 \\
62.88\end{array}$ & $\begin{array}{r}90.90 \\
7.07 \\
1.01 \\
5.05 \\
1.01 \\
2.02 \\
- \\
2.02 \\
72.72\end{array}$ & $\begin{array}{r}81.34 \\
13.46 \\
0.92 \\
5.50 \\
0.61 \\
3.36 \\
1.22 \\
4.28 \\
51.99\end{array}$ & $\begin{array}{r}80.00 \\
12.39 \\
1.15 \\
5.99 \\
0.40 \\
3.37 \\
1.80 \\
3.73 \\
51.17\end{array}$ \\
\hline
\end{tabular}

$x$, DR other than 4,3 or 2

with the colourless soluble substrate tetramethyl benzidine (TMB), which was converted to a blue precipitate by HRP in the presence of hydrogen peroxide. The membranes were transferred into buffer $\mathrm{C}(0.1 \mathrm{~mol} / \mathrm{l}$ sodium citrate, $\mathrm{pH}=5)$ plus $0.1 \mathrm{mg} / \mathrm{ml} \mathrm{TMB}$ from a stock solution of $2 \mathrm{mg} / \mathrm{ml}$ in $100 \%$ ethanol. The detection procedure was performed at room temperature with moderate shaking. Placing the membranes in deionized water helped to intensify signals. For permanent records, the membranes were photographed. Figure 1 presents an example of the hybridization membrane. Initially, the membranes were read independently by two persons (T.B and A. B.).

Risk categorization. Coded results of typing were transferred via Internet to Denver and categorized using a computer algorithm to reduce clerical error. Newborns were categorized into three risk groups (Table 2), with the odds of developing IDDM by the age of 20 years: high $-1: 16$, moderate $-1: 75$ (in non-Hispanic whites) or 1:230 (in Hispanics), or low (less than 1:300).
These risk estimates were calculated using the incidence rates of IDDM reported previously for the Colorado non-Hispanic white and Hispanic population [18] and the HLA-DR, DQ genotype frequency estimates for non-Hispanic white and Hispanic IDDM patients $[13,14,19]$. Such data are not available for Colorado African, Native and Asian Americans; however, these groups account jointly for only $10 \%$ of all children and less than $1 \%$ of IDDM cases in the study population. Children with the high-risk genotype DR3/4, DQB $1 * 0302$ are at about 19-times higher IDDM risk, compared with the general population [14]. Among children assigned to the moderate IDDM risk group, those with DR4/4, DQB $1 * 0302$ are at a sevenfold risk, while those with $\mathrm{DRx} / 4, \mathrm{DQB} 1 * 0302$ or $\mathrm{DR} 3 / 3$ are at a fourfold risk, compared to the general population (where $\mathrm{x}$ is neither DR2, 3 nor 4). Among low-risk children, those with $\mathrm{DR} 3 / \mathrm{x}$ are at risk similar to that for the general population, i.e., 1 in 300 , while those with other genotypes are at a lower risk $[13,14]$. These estimates are based on the distributions of 


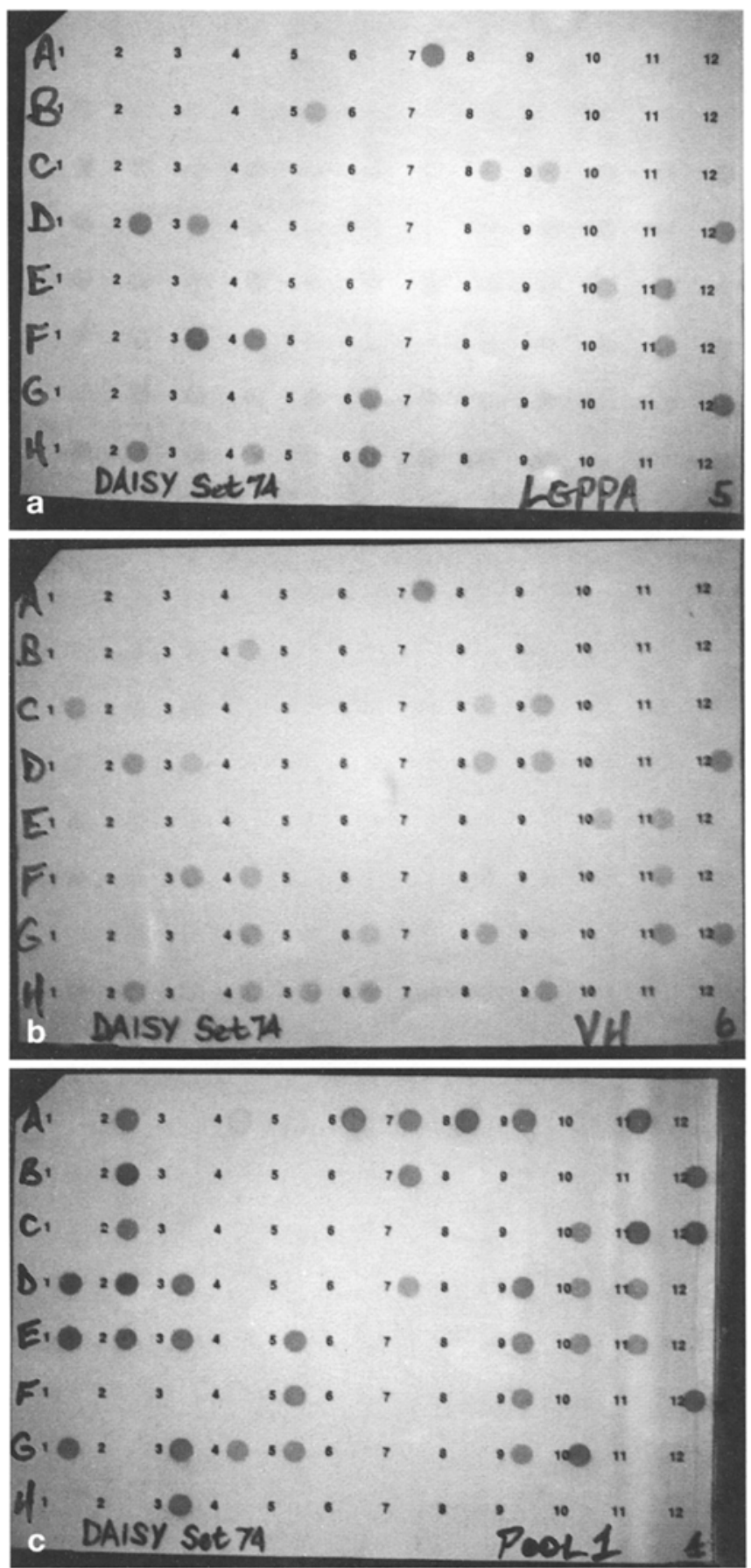

Fig.1 a-c. Representative example of membranes hybridized with labelled individual probes or with pooled probes. a) Immobilized PCR products hybridized with probe DB272 (LGPPA) specific for DQB $1 * 0302 ;$ b) Immobilized PCR products hybridized with the probe GH59 (VH) specific for DRB1*04; c) Immobilized PCR products hybridized with Pool 1, a mixture of probes DB495, GH102, GH111 and CRX24, specific for DR1, DR8, DR12, DR9 and DR10 (see Table 1)

HLA DR and DQ genotypes in patient and control populations reported in the literature; as more data are generated, these estimates should become more precise. High- or moderate-risk children are enrolled in a follow-up study to better understand the natural history of beta-cell autoimmunity and its environmental determinants. Information concerning dietary, infectious diseases and other exposures is collected prospectively by telephone interview. Blood and other samples are obtained for measurements of exposure and islet autoantibodies at the ages of $9,15,24,36,48$, and 60 months.

\section{Statistical analysis}

Population frequencies of HLA genotypes were expressed as percent and compared using test for proportions.

\section{Results}

During the 17 -month period from February 1994 to June 1995, 5053 children were screened. For all except six cases, the HLA-DR,DQ screening category could be unambiguously determined. In these six cases, three alleles could be identified, suggesting contamination with maternal blood. Table 2 summarizes the screening results by ethnicity of the child (the six children with ambiguous typing and two children whose ethnic origin was not recorded were excluded). The frequencies of the high-risk genotype (DRB1*03/DRB1*04, DQB1*0302), were similar among non-Hispanic whites $(2.4 \%)$ and Hispanic whites $(2.8 \%)$, but lower among African Americans $(1.6 \%)$. No child with the high-risk genotype was found among 99 Asian American children. Moderate-risk genotypes were more frequent among Hispanic $(24.4 \%)$ than among non-Hispanic white $(16.7 \%)$, African American $(9.7 \%)$ or Asian American $(9.1 \%)$ children. This was especially true for the DRB1*04, DQB1*0302/x genotype.

The newborns generally did not have a family history of IDDM. Among the 5045 newborns screened, $15(0.3 \%)$ had a diabetic mother, $20(0.4 \%)$ had a diabetic father and $4(0.07 \%)$ had a diabetic sibling. None of the 134 children whose parents refused the screening, but completed the screening interview, had a first-degree relative with IDDM.

The quality control procedures included blinded duplicate HLA typing of the same blood sample for all high- and moderate-risk genotypes and a random $6 \%$ of the low-risk genotypes. We found only four discordant cases and all occurred in the first 10 weeks of the study. None of the 59 repeat typing results of initially high-risk genotypes gave a discordant result. Repeat typing gave discordant results for 2 out of 451 moderate and 2 of the 272 low-risk genotypes. Thus, the reproducibility of the screening was over $99 \%$.

\section{Discussion}

Like many screening approaches, our HLA class II typing strategy represents a trade-off between high 
resolution typing and the need for a rapid, inexpensive, and simple procedure. Thus, we chose not to distinguish the DRB $1{ }^{*} 04$ subtypes, even though the polymorphism at this locus can influence IDDM risk $[13,14,17]$. On DQB1*0302 haplotypes, DRB1 $* 0401$ and *0402 (in Caucasoids) and *0405 (in Caucasoids, Blacks and Asians) confer susceptibility, while *0403 (in Caucasoids and Chinese) and *0406 (in Japanese) are protective and the other alleles appear to be neutral $[14,15]$. We chose to identify the protective DR2 haplotype with a single probe common to all DR2 haplotypes. We do not subtype DR2, even though we are aware that only some subtypes confer protection, because the protective subtypes represent the vast majority of DR2 alleles in the Denver population. Identification of the protective DR2 (DRB1*1501, DQB1*0602) haplotype and distinguishing it from other DR2s would require several probes and a more complex test protocol.

If such a newborn screening programme were to be implemented as a routine procedure, the volume of the blood sample could be easily reduced to 10 $25 \mu \mathrm{l}$ or less and the procedure could be automated. The cost (currently estimated at around U.S. $\$ 10$ $15)$ could be reduced with automation, provided the total test volume was high. Cost-effectiveness considerations might suggest that a simpler test that identifies only the high-risk genotype may be appropriate for some screening.

The PCR-based typing system proved to be highly reliable, with a discordance rate on repeated blinded typing of 782 samples of only $0.5 \%$. Contamination with maternal blood, inferred by the presence of a third allele, clearly interfered with screening in six $(0.1 \%)$ of the cases. There may be undetected cases of maternal blood contamination. For instance, given the nature of our pooled probe typing strategy, if a child were DR1/8 and the mother were DR4/8, and the mother's DNA significantly contaminated the cord blood, it would be typed as DR4/x. Contamination with maternal blood also may not be detectable in the case of homozygosity, e.g. if the child is DR3/3 and the mother is DR3/x their mixed blood would type as DR3/x. Although contamination did not appear to be a significant problem in the screening of cord blood, its exact rate should be determined in future studies by retyping the HLA-DR, DQ genotypes on blood samples obtained directly from the child.

Until recently, non-HLA genetic markers of IDDM risk have not been sufficiently characterized to be included in a screening programme. A few IDDM susceptibility loci have now been mapped outside the HLA region [6-10]. At present, the insulin gene region on chromosome $11 \mathrm{p}[6-8,20]$ is the best-characterized additional marker. Given that the HLA region is, by far, the major genetic determinant of IDDM risk [7, 8], the benefit of adding non-HLA markers remains to be determined. While an effect of non-inherited maternal antigens on IDDM risk has been suggested [21], it has not been confirmed in two large data sets [22], supporting our assumption that genetic risk of IDDM can be assigned on the basis of the child's genotype alone.

In the DAISY project, we will eventually screen 10000 newborns over a period of 3 years; an estimated 31 of these children would be expected to develop IDDM by the age of 20 years. There would be an estimated 12 cases among an estimated 220 highrisk children, an additional 10 cases among an estimated 1000 moderate-risk non-Hispanic white children, 3 cases among an estimated 700 moderate-risk minority children, and 6 cases (5 with DR3/x genotype) among an estimated 8100 low-risk children. This HLA screening identified about $20 \%$ of children from the general Denver population as being at increased IDDM risk. An estimated $80 \%$ of future IDDM cases will occur in this group. An additional $15 \%$ of the future cases will develop among the DR3/x children, representing $13 \%$ of the general population. Thus, at least $30 \%$ of the general population has some degree of genetic predisposition to IDDM, but only about $0.5 \%$ progress to IDDM.

The positive predictive value of this genetic screening, for development of IDDM by the age of 20 years, would be $6.3 \%$ in the high-risk group and between $0.4 \%$ (in non-Hispanic whites) and $0.13 \%$ (in Hispanics) in the moderate-risk group. These estimates are based on genotype frequencies and IDDM incidence estimates reported in the literature and may conceivably need to be revised as more data become available. Subtyping of the DRB $1 * 04$ alleles may increase the positive predictive value of the screening in Hispanics. More Hispanics (27\%), compared with non-Hispanic whites $(19 \%)$ were classified as being at high or moderate risk, while the incidence of IDDM in Hispanics is only $60 \%$ of that in non-Hispanics [18], indicating a lower positive predictive value of this screening for Hispanics. On the other hand, among persons with DR4/DR2, more Hispanics than non-Hispanic whites carry a neutral DR2 haplotype, rather than the protective DR2(DRB1*1501, DQB1*0602) haplotype and are, in fact, at an increased risk of IDDM. Approximately $50 \%$ of Hispanics with the HLA-DR4/2 genotype carry a Native American neutral DR2(DRB1*1602, DQB1*0301) haplotype, compared with 10-15\% of non-Hispanic whites carrying a neutral DR2 (DRB1*1601, DQB1*0502) haplotype. Our screening strategy represents a compromise of cost, sensitivity and positive predictive value for non-Hispanic whites, but more work may be needed to make it equally predictive in other ethnic groups.

Screening for genetic markers associated with (but not diagnostic for) a severe and currently incurable disease, such as IDDM, raises important ethical issues. Our study placed special emphasis on: 1) 
voluntary participation ensured by the informed consent process; 2) full disclosure of the results of the screening to parents, combined with education about IDDM and genetic risk counselling; and 3) confidentiality of genetic information that cannot be disclosed to health providers or other parties without parental consent. The informed consent process for the follow-up phase of the study was separated from the consent to screen and involved additional genetic counselling.

At the present time, such screening appears to be justified only for research purposes. If inexpensive and safe primary prevention of prediabetic autoimmunity becomes available, it could be applied to the general population without genetic screening. However, if the intervention is associated with adverse effects, is expensive or is difficult to execute, genetic screening may be essential to identify those who could actually benefit from the primary intervention. Alternatively, if secondary prevention of IDDM in persons with autoimmunity is successful, general population screening programmes based on repeated autoantibody testing could benefit from population stratification in terms of genetic risk. We have already begun testing the DAISY cohort for beta-cell autoantibodies at the ages of 9 and 15 months.

In summary, it is feasible to screen the general newborn population for IDDM-associated HLA alleles using cord blood and rapid, reproducible and inexpensive PCR-SSO technology. Over $90 \%$ of parents consent to the screening, across various ethnic groups and socioeconomic strata. In the United States population, $2.3 \%$ of children carry the highrisk genotype that is present in $30-40 \%$ of IDDM cases; an additional $18 \%$ carry moderate-risk genotypes that are present in $40-50 \%$ of IDDM cases. Children at such defined risk may benefit from periodic testing for beta-cell autoantibodies and from interventions now being evaluated in clinical trials to prevent IDDM.

Acknowledgements. This study was supported by National Institute of Diabetes and Digestive and Kidney Diseases Grant R01 DK-32493. We thank Ms. S. Tudor, Ms. M. Suk Yu, Ms. C. Uhlhorn, Ms. C.Houghton, Ms. R. Garcia, Mr. D. Perdue, and the personnel of the St. Joseph's Hospital for technical assistance as well as Drs. M. Groshek and E. Gay from Kaiser Permanente Colorado for consulting.

\section{References}

1. Atkinson MA, Mclaren NK (1994) Mechanisms of disease: the pathogenesis of insulin-dependent diabetes mellitus. $\mathbf{N}$ Engl J Med 331: 1428-1436

2. Cudworth AG, Woodrow JC (1975) Evidence for HLAlinked genes in "juvenile" diabetes mellitus. BMJ 2: 133-135

3. Horn G, Bugawan T, Long C, Erlich H (1988) Allelic sequence variation of the HLA-DQ loci: relationship to serology and insulin-dependent diabetes susceptibility. Proc Natl Acad Sci USA 85: 6012-6016
4. Todd JA, Bell JI, McDevitt HO (1987) HLA-DQ $\beta$ genes contribute to susceptibility and resistance to insulin-dependent diabetes mellitus. Nature 329: 599-604

5. Erlich H, Bugawan TL, Scharf S, Nepom GT, Tait B, Griffith RL (1990) HLA-DQ $\beta$ sequence polymorphism and genetic susceptibility to IDDM. Diabetes 39: 96-103

6. Owerbach D, Nerup J (1982) Restriction fragment length polymorphism of the insulin gene in diabetes mellitus. Diabetes 31: 275-277

7. Davies JL, Kawaguchi Y, Bennett ST et al. (1994) A genome-wide search for human type 1 diabetes susceptibility genes. Nature 371: 130-136

8. Hashimoto L, Habita C, Beressi JP et al. (1994) Genetic mapping of a susceptibility locus for insulin-dependent diabetes mellitus on chromosome 11q. Nature 371: 161-164

9. Field LL, Tobias R, Magnus T (1993) A locus on chromosome 15q26 (IDDM3) produces susceptibility to IDDM. Nature Genetics 8: 189-194

10. Owerbach D, Gabbay KH (1995) The HOXD8 locus (2q31) is linked to type I diabetes: interaction with chromosomes 6 and 11 disease susceptibility genes. Diabetes 44: $132-136$

11. Pilcher CC, Dickens K, Elliott RB (1991) ICA only develop in early childhood. Diabetes Res Clin Pract 14 [Suppl 1]: s82

12. Nepom G, Erlich HA (1991) MHC class II molecules and autoimmunity. Ann Rev Immunol 9: 493-525

13. Erlich HA, Rotter JI, Chang J et al. (1993) HLA class II alleles and susceptibility and resistance to insulin-dependent diabetes mellitus in Mexican-American families. Nature Genetics 3: 358-364

14. Rønningen KS, Spurkland A, Tait BD et al. (1992) HLA class II associations in insulin-dependent diabetes mellitus among Blacks, Caucasoids, and Japanese. In: Tsui K, Aizawa M, Sasazuki T (eds) HLA 1991, Vol. 1. Oxford University Press, Oxford, pp 713-722

15. Bugawan TL, Erlich HA (1991) Rapid typing of HLADQB1 DNA polymorphism using non-radioactive oligonucleotide probes and amplified DNA. Immunogenetics 33: $163-170$

16. Erlich HA, Bugawan T, Begovich A et al. (1991) HLA-DR, DQ, and DP typing using PCR amplification and immobilized probes. Eur J Immunogenetics 18: 33-55

17. Van der Auvera B, Van Waeyenberge C, Schuit F et al. and the Belgian Diabetes Registry (1995) DRB1*0403 protects against IDDM in Caucasians with the high-risk heterozygous DQA1*0301-DQB1*0302/DQA1*0501-DQB1*0201 genotype. Diabetes 44: 527-530

18. Gay EC, Hamman RF, Carosone-Link PJ et al. (1989) Colorado IDDM registry: lower incidence of IDDM in Hispanics. Diabetes Care 12: 708-710

19. Cruickshanks KJ, Jobim LF, Lawner-Heavner J et al. (1994) Ethnic differences in human leukocyte antigen markers of susceptibility to IDDM. Diabetes Care 17: 132-137

20. Bennett ST, Lucassen AM, Gough SCL et al. (1995) Susceptibility to human type 1 diabetes at IDDM2 is determined by tandem repeat variation at the insulin gene minisatellite locus. Nature Genetics 9: 284-292

21. Rubinstein P, Ginsberg-Fellner F (1990) The genetics of insulin-dependent diabetes mellitus susceptibility differs among Caucasoids: New York families of Askenazi Jews of Northern European descent. Third International Congress on Jewish Genetic Diseases Tel Aviv, Oxford University Press, Oxford, pp 431-439

22. Bain SC, Rowe BR, Barnett AH, Todd JA (1994) Parental origin of diabetes-associated HLA types in sibling pairs with type I diabetes. Diabetes 43: 1462-1468 